\title{
Fondements théoriques pour une régulation d'Internet : La légitimation faible et la réflexivité forte
}

\author{
Bernard FALLERY \\ Florence RODHAIN \\ Université Montpellier 2 \\ MRM Montpellier Recherche Management \\ Place Eugène Bataillon, 34095 Montpellier
}

\section{Résumé :}

La gouvernance d'Internet arrive au premier plan des préoccupations internationales, mais apparaît aujourd'hui dans une impasse, à la fois au niveau pratique des régulations quotidiennes mais aussi au niveau théorique si elle ne s'appuie que sur une vision contractuelle issue de la Corporate Governance. L'objectif est ici de proposer les fondements théoriques nécessaires pour élaborer des modes alternatifs de régulation de ce réseau de réseaux. La première partie expose un état des forces en présence et des questions posées, en opposant deux visions : celle de la «société de l'information » à celle de «sociétés du savoir ». Dans une deuxième partie on présente alors une proposition théorique fondée sur deux concepts issus des recherches sociologiques : la légitimation faible et la réflexivité forte. Au final il apparaît que l'évolution vers les deux scénarios possibles, une démocratie directe sans hiérarchie ou une démocratie représentative ordonnée, passera par une même culture intermédiaire, celle de la solidarité et de la sécurité collective. La discipline des Systèmes d'information doit apporter son éclairage sur les conditions de cet apprentissage inter-organisationnel.

Mots clés : Internet, Gouvernance, Régulation, Légitimation, Réflexivité

\begin{abstract}
:
Internet governance represents a hot issue, difficult to approach, both in practice of daily regulation and in theory if it is only based on the Corporate Governance vision. The objective of this article is to search for theoretical foundations in order to provide alternative regulation of this network of networks. The first part describes a state of forces and issues raised, between two visions: "the information society" and "the knowledge societies". A second section presents a theoretical framework based on two concepts from sociological research: legitimacy and reflectivity. As a result it appears that the move to two possible scenarios, a direct democracy or a representative democracy, pass through a newculture, that of solidarity and collective security. The discipline of information systems should provide insight into the conditions of this inter-organizational learning.
\end{abstract}

Key words: Internet, Governance, Regulation, Legitimacy, Reflexivity 


\section{Introduction}

Qui gère Internet ? Comment gouverner sans gouvernement ? Le concept de « gouvernance » d'Internet arrive au premier plan des préoccupations internationales. Mais cette gouvernance apparaît aujourd'hui dans une impasse, à la fois au niveau pratique dans les régulations quotidiennes (l'impossible contrôle du pourriel, spam, est un bon exemple ${ }^{1}$, les difficiles débats sur la gestion des noms de domaines ou le contrôle des contenus en sont d'autres...), mais aussi au niveau théorique si elle ne devait s'appuyer que sur la vision contractuelle issue de la Corporate Governance. Notre objectif est alors double pour la discipline des Systèmes d'information: montrer d'une part la contribution qu'elle peut aujourd'hui apporter dans la définition des problèmes et le choix de nouveaux fondements théoriques, et l'inciter d'autre part à améliorer cette contribution en s'appuyant sur ses apports distinctifs dans le domaine des TIC : coordination, confiance, usages, performance, connaissances, apprentissage organisationnel et inter-organisationnel...

La popularité grandissante du terme de gouvernance en Gestion comme en Economie ne facilite pas les analyses : on l'emploie de plus en plus abusivement pour désigner soit l'ensemble de la gestion (science de la décision rationnelle), soit même l'ensemble du management (pilotage des activités, développement des structures, conduite des hommes dans les organisations) soit même encore l'ensemble du développement économique (avec l'idée que l'environnement institutionnel est devenu un déterminant crucial de ce développement). Après tout, cela n'est peut-être pas si gênant diraient certains, mais à un (gros) détail près. Les fondements théoriques de la gouvernance sont en effet trop exclusivement liés à la «Corporate Governance » et notamment à l'article de Jensen et Meckling publié en 1976: une théorie de l'agence pour expliquer la structure de financement et la structure de propriété, et une focalisation sur les systèmes d'incitation et d'évaluation de la performance financière (Charreaux et Schatt 2005). Selon le mot de Bournois et al. (2007) il s'agit d'ailleurs plutôt ici de « dirigeance » que de gouvernance.

Et même si on tient compte de l'élargissement de cette théorie au départ purement financière et actionnariale (encadrement des conflits entre dirigeants et investisseurs, mécanismes de distribution de la richesse en faveur des mandants de l'entreprise, EVA Economic Value Added, stock options, marché des dirigeants...) à une théorie plus partenariale de la gouvernance (qui reconnaît la pluralité des objectifs et qui associe de multiples parties prenantes détenant des droits et des devoirs légitimes : salariés, clients, fournisseurs, sous-traitants... syndicats, citoyens, ONG), cela n'en change pas

\footnotetext{
${ }^{1}$ http://lesrapports.ladocumentationfrancaise.fr/BRP/044000022/0000.pdf Rapport de mission J.M Yolin 2004. Des données différentes circulent, mais le pourriel non sollicité (le plus souvent à finalité commerciale) représenterait plus de $70 \%$ de tous les courriels envoyés. Ceci expliquerait notamment la faible utilisation du courriel sur les liaisons à bas débit des zones les moins développées. Une «simple » régulation d'ordre technique serait certes entre les mains des quelques détenteurs de bande passante... mais avec un risque non accepté de domination par le contrôle sur les contenus, et avec une remise en cause du principe de séparation entre les infrastructures et les contenus.
} 
vraiment le fondement théorique (Martinet 2008, Pesqueux 2007). Il s'agit toujours d'une vision contractuelle individualiste, où les notions de coûts contractuels et de « partenariats public-privé » viennent certes élargir considérablement la notion de coût d'agence, mais :

- qui pré-suppose toujours l'optimalité,

- qui met toujours en avant la réduction de coûts, mais sans tenir compte des externalités (notamment celles inhérentes aux inégalités générées par la dynamique des marchés, Pérez 2003),

- qui néglige l'ensemble des pressions réglementaires, politiques, technologiques et environnementales (Rodhain et Fallery 2008),

- enfin qui n'intègre aucune vison historique ni même dynamique de la construction collective des interactions et des institutions (Pesqueux 2008).

On peut d'ailleurs noter que la question très générale de la gouvernance est bien antérieure à celle de la gouvernance des entreprises : elle est centrale en science politique depuis le concept de séparation des pouvoirs, comme est elle centrale en droit pour analyser la « juridiciarisation » des sociétés contemporaines et en économie autour des systèmes de co-régulation depuis la faillite des modèles uniquement centrés sur l'Etat (Lenoble 2005). Comme le propose Pesqueux (2008) dans sa critique de la Corporate Governance notre responsabilité serait d'assurer le passage d'une gouvernance restreinte (vue comme ontologie de l'entreprise) à la gouvernance élargie (vue comme ontologie de l'organisation).

Pour Internet, le terme de gouvernance a pourtant été consacré lors du SMSI (Sommet mondial de la société de l'information) en 2005 par l'Agenda de Tunis §34, qui le définit comme « l'élaboration et l'application par les Etats, le secteur privé et la société civile, chacun selon son rôle, de principes, normes, règles, procédures de décision et programmes communs propres à modeler l'évolution et l'utilisation de l'Internet $»^{2}$. Lors de ce sommet, le secrétaire général Kofi Anann a notamment déclaré : «L'ONU ne souhaite pas prendre le contrôle de l'Internet, le policer ou le réglementer de toute autre manière... Le but commun que nous visons, c'est de protéger et de consolider l'Internet et de faire en sorte que tous puissent en bénéficier. Les États-Unis méritent notre gratitude pour avoir développé l'Internet et l'avoir mis à la disposition du monde entier... Mais je pense que vous serez également unanimes à reconnaître la nécessité d'une plus grande participation internationale aux débats sur les questions relatives à la gouvernance de l'Internet. Le problème est de savoir comment cet objectif pourra être atteint ».

Le décor est ainsi planté, et les trois grandes questions sont posées :

\footnotetext{
${ }^{2}$ http://smsi.internet.gouv.fr
} 
- comment faire bénéficier d'Internet au plus grand nombre ? (la culture et le savoir),

- comment protéger à la fois le réseau, les états et les utilisateurs (la liberté et la surveillance),

- comment organiser la régulation d'Internet ? (la gouvernance).

Mais derrière ces trois questions se profilent au moins deux grandes visions bien différentes, s'abritant soit derrière un discours plutôt technique, soit derrière un discours plutôt social : déréglementation et/ou exception culturelle ? Surveillance de la criminalité et/ou liberté d'expression? Droits de propriété et/ou biens publics mondiaux ? Normalisation par des experts et/ou régulation par les élus ? Marché de la connaissance et/ou communautés locales? Si tant de gens réfléchissent à "l'Internet du futur", c'est bien que celui-ci va rencontrer des problèmes, il est important d'en mesurer le caractère plus ou moins critique. La première partie de notre proposition expose donc un état des forces en présence et des questions posées, en opposant deux grandes visions d'Internet : celle de «la société de l'information » et celle «des sociétés du savoir».

Notre proposition est alors que l'on ne s'accordera pas sur la régulation d'Internet tant que les fondements théoriques ne dépasseront pas la vision contractuelle héritée de la Corporate Governance. Le recours presque systématique au concept de gouvernance, en économie comme en gestion, s'explique à notre avis par l'insuffisance des fondements théoriques standards (l'économie comme agrégation des préférences, ou l'économie comme nœuds de contrats) pour décrire une société où apparaissent en fait de nouveaux types de «biens communs mondiaux ${ }^{3}$ et où les rapports sociaux approfondissent à la fois l'individualisme et la socialisation. L'objectif de la deuxième partie est donc de partir à la recherche de nouvelles bases théoriques de la régulation, dans la perspective ouverte par certains travaux sur la légitimation faible (Habermas 2000, Foucault 1978) et sur la réflexivité forte (Bourdieu et Wacquant 1992, Beck, Giddens et Lash 1994).

\section{Deux visions pour Internet : celle de « la » société de l'information ou celle « des » sociétés du savoir}

La discipline des Systèmes d'Information a longuement débattu du déterminisme technologique, du déterminisme organisationnel et du concept d'émergence. Ce débat permet de mettre en lumière deux approches bien différentes du «phénomène » Internet : une approche plus technique et déterministe (1.1.: la vision de l'UIT, Union Internationale des Télécommunications, pour une «société de l'information») et une approche plus sociale et culturelle (1.2. : la vision de l'UNESCO pour des « sociétés du savoir »).

\footnotetext{
${ }^{3}$ http://www.coll.mpg.de/ Max Planck Institut for research of collective goods
} 
Le concept de «société de l'information »a été préparé depuis la fin de la deuxième guerre mondiale par tout un travail de fond de militaires, de scientifiques, d'industriels et d'intellectuels (Mattelard 2003), et il a acquis aujourd'hui un caractère d'évidence dans les organisations internationales, sans véritable débat. Le démantèlement d'ATT en 1984 par l'administration Reagan donne le coup d'envoi aux réseaux transfrontières et à la déréglementation des services publics. En 1998, l'accord de l'OMC consacre l'ouverture des marchés des télécoms. En 1994, le projet d'autoroutes globales de l'information est lancé par l'administration Clinton, et en 2000 le sommet du G8 à Okinawa lance finalement une charte de la « société globale de l'information » (même si le tiers de l'humanité ne dispose toujours pas d'électricité...).

Le concept de « sociétés du savoir » aurait pu être au cœur du SMSI, à Genève en 2003 puis à Tunis en 2005. Ce somment pouvait apparaître comme le lieu d'un premier débat sur la ou les sociétés de l'information, en réunissant les gouvernements, les organisations intergouvernementales de l'ONU, les ONG de la société civile et le secteur privé (qui a obtenu à cette occasion, et pour la première fois, un statut de quasi-ONG). Mais, à part la fixation de vastes objectifs pour 2015 et la mise en place du Forum annuel sur la gouvernance d'Internet IGF et du Forum annuel de suivi SMSI ${ }^{4}$, le principal résultat du Sommet de Tunis aura plutôt été de ne pas résoudre les deux questions brûlantes du moment : celle du financement du Fond de Solidarité Numérique et celle de la succession de l'ICANN (Internet Corporation for Assigned Names and Numbers) la société californienne en charge de la gestion des noms de domaines.

\subsection{La vision technique et déterministe de la « société de l'information »}

L’UIT peut être considérée comme un bon représentant de la vision technique de «la » société de l'information, autour de maîtres mots comme: Autoroutes de l'information, Nouvelle économie, Fracture numérique, Globalisation, Logique de l'accès, Marchandisation, Déréglementation... c'est le mythe du progrès.

\subsubsection{L’UIT, comme représentant de la vision technique d'Internet}

«Lorsque chacun pourra communiquer en utilisant son téléphone traducteur, il y aura moins de guerres qui génèrent la pauvreté, et peut-être même que la paix éclatera dans le monde ».

(Yoshiro Utsumi, Secrétaire général de l’UIT, dans le Financial Times, 8 octobre 1999).

\footnotetext{
${ }^{4}$ www.intgovforum.org et www.wsis-communauty.org Le WGIG est le Groupe de travail sur la gouvernance de l'Internet, créé par l'ONU en 2003.
} 
«La libéralisation des télécommunications sur le continent africain commence à porter ses fruits. Et il faut tenir compte aussi du merveilleux cadeau qu'ont reçu les habitants de ce continent avec l'avènement du cellulaire mobile et des cartes à prépaiement ».

(dans Nouvelles de l’UIT n9/2001).

Rappelons pourtant qu'à cette époque en 2001 ce «merveilleux cadeau » ne représentait en moyenne que 1,2 lignes/100 habitants et que toute l'Afrique subsaharienne ne comptait que 1,3 millions d'utilisateurs d'Internet (source http://csdptt.org/).

L'UIT, désignée comme coordonnatrice et organisatrice du SMSI, est une institution publique intergouvernementale qui, à la différence des autres organisations des Nations unies, ne regroupe que deux catégories de membres officiels: les états et le secteur privé (opérateurs, constructeurs, prestataires de services, organismes de recherche, organisations internationales de financement et de développement... avec Cisco et Microsoft bien sûr, mais aussi des partenaires plus douteux comme Eron, WorldCom, Qwest, Global Crossing, Tyco...). Etant la plus ancienne organisation internationale technique de coordination, puisqu'elle a été créée en 1865 sous le nom d'Union internationale du télégraphe, elle bénéficie d'une grande légitimité pour élaborer la réglementation, la normalisation, la coordination et le développement du secteur des télécommunications internationales. Mais l'UIT, pourtant agence des Nations Unies depuis 1947, ne reconnaît pas les organisations ONG de la société civile: ses membres ne sont que des états et des entreprises privées, soumis au paiement d'une cotisation qu'ils choisissent suivant leur classe de contribution. Son activité est exercée par trois entités appelées Secteurs : celui des radiocommunications, celui de la normalisation des télécommunications et celui du développement des télécommunications. Mais c'est dans les Groupes (d'Etudes, de Travail, Spécialisés...) que se dessine la stratégie d'évolution des technologies. Suite à une grave crise de financement, le personnel de l'UIT est passé de plus de mille agents en 2000, à moins de 800 à la fin de 2003. L'UIT espère que son expérience dans le domaine de la normalisation justifiera des responsabilités étendues à l'Internet dans le cadre de la réflexion en cours, mais la concurrence est rude...

Le spectre des fréquences et les orbites satellitaires sont des ressources limitées qui doivent donc être gérées, et elles devraient normalement relever d'un patrimoine mondial, comme l'air ou les océans. Sous la pression de l'OMC et de l'UIT, le dogme des «règles » du marché en a fait des marchandises et a créé de belles rentes de situation (Rioux 2004). La déréglementation est devenue le

\footnotetext{
${ }^{5}$ L'article "A Brief History of the Internet" de l'ISOC présente l'évolution historique de la normalisation d'Internet. Les principaux organismes actuels sont l'IAB (Internet Architecture Board), l'IETF (Internet Engineering Task Force), l'IRTF (Internet Research Task Force) et le W3C (World Wide Web Consortium). http://www.isoc.org/graphics/timeline.gif
} 
concept de base de l'UIT, même pour le développement des réseaux de télécommunications du «Sud » (sources Benamrane et al. 2005, Fullsack J.L http://csdptt.org/ ) :

- $\quad$ ouverture « urgente » à la concurrence jusque dans les PMA, Pays les Moins Avancés... ce qui a permis une reprise à bon marché des opérateurs publics par les sociétés des pays riches ;

- engagement financier de l'UIT en faveur des «Académies Cisco » et d'un réseau de gros routeurs « Tout IP » basés sur les produits Cisco ( $85 \%$ des parts du marché mondial)... ce qui a concurrencé les fragiles universités africaines en informatique et aussi entraîné une véritable coupure dans l'évolution des réseaux africains ;

- attribution de la bande de $2 \mathrm{GHz}$ aux radiocommunications mobiles y compris satellitaires (pour Iridium, ICO, Teledesic... aujourd'hui disparues)... ce qui a mis dans une position critique les réseaux fixes terrestres en Afrique ;

- projets d'infrastructures contradictoires entre le projet AFRICA ONE (une boucle de $35000 \mathrm{~km}$ de câbles optiques sous-marins qui devait entourer l'Afrique et connecter une trentaine de villes portuaires : un projet de 2 milliards de \$ lancé dès 1994 dans un partenariat ATT-UIT ... et finalement annoncé comme « mort-né » à la veille du SMSI en 2005, en engloutissant des dizaines de millions de dollars) et le projet RASCOM (un projet de 400 millions de dollars pour une couverture par satellites, mais qui n'a pas encore tout son financement et subit de nombreux déboires....). ${ }^{6}$

Les travaux de préparation et de tenue du SMSI ont été confiés par l'ONU à l'UIT, institution à vocation technique qui milite pour le libéralisme en matière de télécommunications. On est en droit de se demander si le SMSI aurait travaillé avec les mêmes approches ce sommet avait été confié à l'Unesco, agence de l'ONU compétente pour «l'information et pour la communication», plus politique, sensible au respect des droits humains, aux impératifs de culture et de coopération, et moins axée sur les intérêts des opérateurs privés et le libre exercice des règles du marché.

\subsubsection{Des propositions « techniques » pour des questions ... très politiques.}

Bien au-delà de l'UIT, cette vision technique de la société de l'information est bien sûr celle des sociétés multinationales mais aussi celle des gouvernements, pour résoudre certains problèmes

\footnotetext{
${ }^{6}$ Du fait de la facturation des accords de «peering » entre le Nord et le Sud, il revient trois fois plus cher de se connecter à l'internet au Sud plutôt qu'au Nord... (Jean-Michel Cornu)

http://www.correspondants.org/news/les-accords-de-peering-ou-comment-le-sud-finance-le-nord
} 
jugés aujourd'hui sensibles, notamment la gestion des noms de domaines, la tarification étagée et le contrôle des contenus.

La gestion ou le monopole des noms de domaines? Le contrôle des noms de domaine (DNS Domain Name System) est géré depuis 1998 par la société californienne (ICANN) sans actionnaire ni assemblée générale, et où le Département du Commerce étasunien dispose d'un droit de veto ${ }^{7}$ (Brousseau, 2001). Pour d'évidentes raisons techniques, l'arbre des noms de domaine avait été organisé dès le départ de manière centralisée par Jon Postel, professeur à l'UCLA : il y a aujourd'hui treize serveurs racines gérant la base DNS, dont le serveur de tête géré par la société Verisign. L'ensemble des routeurs du monde (gérés eux par la société Cisco) vient copier sur ces treize serveurs racines les arbres de nommage qui s'y trouvent. La conversion des adresses IP numériques par pays ou par genre (dont les .com, .net, .org qui sont gérés aussi par la société Verisign) permettrait de rendre inopérantes les ressources d'un pays tout entier (et il semble que ce fut le cas une fois pour la Libye). À ce titre, les Etats-Unis considèrent comme «non souhaitable » (!) une gestion multilatérale de ces ressources $^{8}$ (un veto a par exemple été opposé par l'administration Bush à la création, pourtant voulue par l'ICANN, d'un domaine .xxx réservé aux sites pour adultes). Ce problème de la gestion des adresses va prendre bientôt toute son ampleur avec «l'Internet des objets » et les systèmes de traçabilité. Avec le remplacement des codes barres par des étiquettes radiofréquence intelligentes et avec le passage du protocole IP de la version 4 à la version 6, l'ensemble des biens et marchandises sera progressivement connecté à l'Internet via la technologie ONS Object Naming Service ${ }^{9}$. Une liberté fondamentale pour les citoyens sera bientôt de pouvoir établir un périmètre de sécurité autour

\footnotetext{
${ }^{7}$ Les relations entre l'ICANN et le Ministère du Commerce des Etats-Unis ont été clarifié en septembre 2009, par les signatures conjointes d'une affirmation d'engagements ( « un pas en avant en termes de progrès et d'internationalisation du modèle de l'ICANN », selon Rod Beckstrom CEO de l'ICANN) http://www.icann.org/fr/announcements/announcement-30sep09-fr.htm

${ }^{8}$ http://www.dnsnews.fr/ Le système de nommage est pourtant passé du simple stade technique à celui d'un véritable marché de 130 millions de noms (avec son «second marché » juteux, lié à la pénurie des adresses, aux conflits de propriété, à la publicité des marques).

L'Union Européenne a dû longuement négocier pour obtenir enfin en 2006 un nom de domaine propre à l'Union. L'opération du «.cn à un yuan» en 2007 a permis de faire passer le nombre de domaines chinois de 2 à 5 millions en trois mois, avec la volonté évidente de pouvoir se détacher du .com. Et l'ICANN tardant à mettre en place un système globalement multilingue, la Chine vient de modifier la structure de son système d'adressage : les utilisateurs peuvent ainsi taper les adresses en .com avec des idéogrammes. Certains y voient une autre manœuvre pour amener les internautes chinois à ne plus passer par les serveurs américains.

Le nouveau protocole IP V6 lèvera la contrainte de rareté sur les adresses numériques nécessaires à l'Internet des Objets (il y a aujourd'hui 10 milliards d'adresses possibles soit l'équivalant d'une par homme, on passera à l'équivalent d'une adresse par atome !), mais le nombre de mots du langage naturel à partir desquelles il est possible de créer des adresses signifiantes est limité, ce qui continuera à poser d'importants problèmes de conflit d'intérêt.

${ }^{9}$ http://www.amazon.fr/gp/reader/0321384016/ref=sib_dp_pt\#reader-page : A. Greenfield parle de 1' «Everyware ». Le tracking géo-logistique (localiser un produit, un dossier ou une personne) et le tracing stratégique (reconstituer un historique, une origine, une activité, un contrôle...) seront alors possibles en permanence sur Internet. La Commission Européenne a organisé en 2006 une consultation en ligne sur les usages liés aux puces à radiofréquence.
} 
d'eux, une sorte de droit au silence. S'il devient possible de suivre les mouvements de tous les objets et personnes sur la planète, le gouvernement qui contrôlera ce système détiendra un pouvoir dont personne n'osait rêver. Et c'est la société Verisign, société sous contrat avec le département étasunien de la défense, qui vient d'être chargée par EPC Global, le consortium mondial des codes barre, d'organiser les règles de ce futur registre ONS.

La neutralité du réseau ou la tarification étagée ? L'architecture technique du réseau « endto-end», où aucun traitement n'est centralisé, avait jusqu'ici élevé au rang de grand principe, celui de « la neutralité d'Internet »(Benhamou 2007, Peha et al. 2007)). Certains ont pu parler ici de «Premier amendement d'Internet » : l'absence de discrimination dans l'accès à toutes les ressources du réseau (les applications, les services, comme les contenus: Web 2.0, peer-to-peer, Blogs...). Mais aujourd'hui l'idéologie libertaire des créateurs d'Internet est bien loin du débat qui fait rage à propos de la «tarification étagée »(tiered Internet), notamment dans la perspective de la télévision numérique: les opérateurs télécoms réclament une tarification spécifique pour les gros utilisateurs comme Google, Amazon ou Yahoo, afin qu'ils puissent bénéficier d'une bande passante protégée, surpayée par les usagers de leurs services particuliers ${ }^{10}$. Cette tarification du débit (à l'arrivée) serait une révolution qui poserait des problèmes importants à certains usages sociaux d'Internet comme les jeux vidéos, les échanges de fichiers ou même l'encyclopédie Wikipédia (laquelle utilise peu de bande passante, mais a pourtant atteint des niveaux équivalents à ceux d'e-Bay ou d'Amazon, ce qui l'oblige déjà à utiliser les infrastructures de Yahoo, pour le moment sans contrepartie commerciale (Sources Internet 2010, en bibliographie)). Les opérateurs privés, et les usagers qui sont aujourd'hui prêts à payer pour bénéficier d'un accès prioritaire à certains services, remettent ainsi en cause un principe fondamental de la Netiquette, celui qui consistait à économiser les ressources rares comme la bande passante.

La liberté ou le contrôle des contenus ? Face aux débordements de plus en plus fréquents du droit conventionnel des Etats ${ }^{11}$, la solution de plus en plus avancée par les industriels et les gouvernements est présentée comme technique : elle repose sur la notion « d'hébergement abusif » en rendant responsables les FAI (Fournisseurs d'Accès à Internet) et les intermédiaires techniques des infractions commises par leurs abonnés, et notamment le «piratage ». Au nom du principe de liberté,

\footnotetext{
${ }^{10}$ www.netgouvernance.org le site de B. Benhamou

${ }_{11}$ http://www.droit-technologie.org/ L'approche étasunienne de la liberté d'opinion est fondée sur le célèbre Premier amendement « Le Congrès ne pourra faire aucune loi ... restreignant la liberté de parole ou de la presse, ou touchant au droit des citoyens de s'assembler paisiblement et d'adresser des pétitions au gouvernement » (1791). L'idée d'une protection du pluralisme par un « libre marché des idées » est confirmée par un arrêt fameux du juge Holmes en 1919, selon lequel «le meilleur test de la véracité d'une idée, c'est sa capacité à se faire accepter au milieu de la compétition du marché », ce qui oblige aujourd'hui à tolérer la propagande néonazie sur Internet tant qu'elle ne menace pas une personne particulière.

L'approche européenne de la liberté est plus fondée sur les dangers de l'abus de pouvoir et l'idée de protection des individus par la loi (Pacte relatif aux Droits Civils et Politiques, Convention Européenne des Droits de l'Homme...). La première directive du Parlement européen et du Conseil concernant la vie privée dans le secteur des télécommunications date de 1995, elle a été souvent actualisée (2000, 2002, 2006).
} 
ce contrôle des contenus par les opérateurs avait pourtant toujours été refusé sur Internet., même pour résoudre le grave problème du pourriel.

Les votes en 2001 puis 2003 des « Patriot Act » aux Etats-Unis ${ }^{12}$ autorisent le FBI à mettre sur écoute et surveiller les FAI sans contrôle judiciaire (avec le logiciel « Carnivore »... il fallait oser un tel sigle !). En Chine un nouveau pas avait été franchi en 2007: les hébergeurs chinois, mais aussi internationaux, ont du signer un «pacte d'autodiscipline» à travers lequel ils s'engagent à ne pas diffuser des messages illégaux et à «protéger les intérêts de l'Etat et du public chinois » en identifiant les blogueurs ${ }^{13}$. En France en 2009, pour satisfaire la demande pressante des industries culturelles, la loi Création et Internet a créé la haute autorité Hadopi : avant sa censure par le Conseil constitutionnel, il s'agissait de permettre aux FAI de couper les connexions sans passer par une autorité judiciaire. En 2010 un accord commercial de lutte contre la contre-façon se négocie secrètement autour de l'Union Européenne et de l'ALENA (et en dehors de toute instance officielle telle que l'OMPI, l'organisation mondiale de la propriété intellectuelle) : l'ACTA, Anti-counterfering Trade Agrement ${ }^{14}$ cherche à s'appuyer sur les FAI pour imposer une protection accrue de la propriété intellectuelle et des brevets de toutes sortes.

Quels que soient les contenus à contrôler (propagande, terrorisme, brevets, droits d'auteur...) on voit que les nouvelles propositions avancées sont loin de n'être que « techniques ».

\subsection{La vision sociale et culturelle des « sociétés du savoir »}

L'UNESCO peut être considéré comme un des lieux où se construit une vision culturelle « $d e s$ » Sociétés du savoir (un concept avancé face à celui «d'une » Société de l'information) ${ }^{15}$, autour des maîtres mots : Diversité culturelle, Multilinguisme, Patrimoine numérique, Logiciels libres, Vie privée, Propriété intellectuelle, Education, Citoyenneté, Liberté d'expression... c'est le mythe du partage.

\footnotetext{
${ }^{12}$ http://www.assemblee-nationale.fr/rap-info/i2623.asp Rapport Paecht, 2000. Le grand principe étasunien du Premier amendement et du marché des idées n'avait pas empêché, ni la chasse aux sorcières dans les années 1940, ni le programme d'espionnage généralisé ECHELON (poursuite depuis 1946 de l'alliance UKUSA entre la NSA et les Alliés anglo-saxons).

${ }^{13}$ http://www.rsf.org/ Reporters Sans Frontières estime que plus de 50 « cyber-dissidents » sont emprisonnés en Chine, souvent accusés d'avoir révélé des secrets d'Etat. Et les autorités chinoises, soucieuses de mieux contrôler les connexions sans fil sur leur territoire, avaient demandé à des industriels américains de modifier les technologies Wi-Fi afin qu'en temps réel le ministère de l'intérieur soit informé de l'identité des personnes connectées. Les industriels américains n'ont pas accédé à la demande des autorités chinoises.

${ }^{14}$ Voir le site de M. Geist, professeur de droit à l'université d'Ottawa www.michaelgeist.ca

${ }^{15}$ Si L'UNESCO cherche à promouvoir des « sociétés du savoir », l'Union Européenne met en avant une «Economie de la connaissance » (pour une analyse critique de ce dernier concept voir « La marchandisation du savoir, Processus de Bologne, Stratégie de Lisbonne » Réseau Europe, www.reseau-europe.eu )
} 


\subsubsection{L'Unesco, comme représentant de la vision sociale et culturelle d'Internet}

Selon l'article 19 de la Déclaration Universelle des Droits de l'Homme de l'ONU, «Toute personne a droit à la liberté d'opinion et d'expression, ce droit englobe la liberté d'avoir ses propres opinions et de chercher, recevoir et partager des informations et des idées à travers tout média sans distinction de pays ».

L'Unesco est l'agence de l'ONU compétente pour « l'information et pour la communication ». Mais dès 1985 le projet des pays non-alignés de définir un «nouvel ordre mondial de l'information et de la communication» (NOMIC) avait déclenché le départ immédiat des USA. Les Etats-Unis sont finalement redevenus membres de l'Unesco depuis 2003 au prix d'une rectification des objectifs stratégiques de l'organisation, notamment l'abandon de ses activités en faveur du logiciel libre et une nette réduction de son budget (au profit de Partenariats Public-Privé PPP, directement financés et gérés par des États membres ou des entreprises multinationales dont Microsoft).

Ces abandons n'ont pas empêché l'adoption en 2005, et malgré l'opposition des USA, de la «Convention pour la protection et la promotion de la diversité des expressions culturelles», aujourd'hui ratifiée par plus de 50 états, avec son article 20 : «lorsqu'elles souscrivent à d'autres obligations internationales, les Parties prennent en compte les dispositions pertinentes de la présente Convention ». Bien que la Cour internationale de justice ne puisse probablement pas statuer (elle est bien citée dans cette Convention Unesco, mais sa consultation n'est pas mentionnée à l'OMC, qui n'est pas une organisation des Nations Unies), l'Unesco devrait donc quand même disposer à terme d'un dispositif «normatif » pour accomplir la mission conférée par son Acte Constitutif de « respecter la féconde diversité des cultures » et de «faciliter la libre circulation des idées par le mot et par l'image ».

Pour l'Unesco « la gouvernance électronique est l'utilisation, par le secteur public, des TIC dans le but d'améliorer l'information et la prestation des services, d'encourager le citoyen à s'impliquer dans le processus des prises de décision et de rendre le gouvernement plus accessible, plus transparent et plus efficace ... C'est au niveau local municipal que l'impact des TIC sur les relations entre les gouvernements et les citoyens, peut être le plus efficace $»^{16}$.

\subsubsection{Des propositions politiques pour des questions... de société}

Personne ne serait bien sûr légitime pour dresser un éventuel ordre de priorité entre liberté d'information, respect de la propriété intellectuelle, respect des identités, multilinguisme, accès universel, lutte contre la pauvreté... Mais la diversité culturelle est avancée comme un concept de base

${ }^{16}$ www.unesco.org Renforcement des capacités pour l'e-gouvernance 2007 
pour l'Unesco, avec au moins trois grands dossiers: celui de l'exception culturelle, celui de la propriété intellectuelle et celui de la fracture numérique.

La standardisation nécessaire ou l'exception culturelle ? Il s'agit d'un dossier où le rôle de la France et du Canada a été important (Marzouki et al. 2004b) dans la formulation d'une doctrine de l'« exception » et dans l'obtention d'un sursis. «Les biens et services culturels, des marchandises pas comme les autres », tel est le titre de l'article 8 de la Déclaration internationale sur la diversité culturelle) face au principe général des «offres de libéralisation» de l'OMC (pour qui, à l'inverse, «La promotion de contenus locaux ne doit pas engendrer des barrières irraisonnables au commerce»). Les questions urgentes concernent notamment le multilinguisme sur Internet ${ }^{17}$ (deux langues et leur culture disparaissent chaque année, sur les 6000 qui restent encore dans le monde), et l'enseignement sur Internet (comment articuler de nécessaires normes d'interopérabilité avec d'indispensables normes «régionales»?). La question du multilinguisme est notamment débattue au Forum sur la

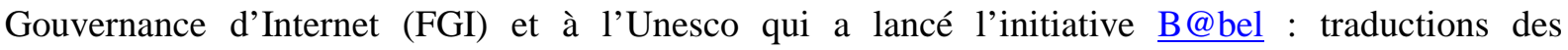
terminologies, recherches sur les langues sur Internet, dictionnaires, polices, formats d'échange, scripts des noms de domaines... La question de l'enseignement en ligne est débattue aussi bien dans les organismes internationaux de normalisation (ISO, ANSI, CEN, AFNOR, DIN...) que dans des associations comme SCORM, IEE, IMS... Par exemple l'IMS, Instructional Modeling System, est un consortium qui regroupe 250 institutions, agences gouvernementales et entités commerciales (dont British Telecom, l'Université de Barcelone, Microsoft...). L'IMS a pris en charge la définition de Learning Design. Ce standard permet de décrire un véritable scénario pédagogique «structuré et réutilisable »donnant tous les détails permettant de réaliser une formation, avec des ressources décrites au standard LOM Learning Object Model adopté par l'ISO et des interfaces décrites au standard SCORM Sharable Content Object Reference Model soutenu par l'initiative américaine ADL. Mais il est clair que cette standardisation, certes nécessaire pour réutiliser des ressources, risque d'imposer un modèle pédagogique béhavioriste très particulier (Fallery, 2004).

La propriété intellectuelle ou l'accès au savoir ? Il s'agit d'un dossier complexe, car les ressources informationnelles sur Internet ne peuvent faire l'objet ni d'une définition précise des droits de propriété (cela va des théorèmes mathématiques ... jusqu'aux informations personnelles sur les individus), ni de règles précises sur les droits d'usage de ces informations (droit d'exclusivité, usage privé ou droit de revendre, copie privée, droit de combiner des informations...) (Vivant 2000, Mueller 2008). Les deux questions urgentes concernent notamment celle des brevets sur la connaissance (propriété industrielle et/ou logiciels libres: licence $G N U G P L$ ) et celle des redevances sur les

\footnotetext{
${ }^{17} 11 \%$ des pages Web sont en langues latines (espagnol, roumain, portugais, français, italien) contre $72 \%$ en anglais, alors que l'ensemble des locuteurs de langues latines dépasse 780 millions contre 630 millions pour les locuteurs de langue anglaise http://unesdoc.unesco.org. 90\% des contenus sur Internet ne sont disponibles que dans 12 langues.
} 
créations (propriété littéraire et artistique : licence Creative Commons, DRM digital right management systems, droit des diffuseurs... $)^{18}$. Cet équilibre instable entre une vision strictement économique de la propriété intellectuelle (considérée par les détenteurs de portefeuilles de brevets comme un droit naturel à l'exemple de la propriété du capital) et une vision sociale du partage du savoir (considérée comme le droit de la société à utiliser la connaissance et les « biens publics mondiaux ») n'a même pas été abordé au SMSI. La pression des états et des entreprises a réussi à renvoyer ces questions à l'OMPI, organisation mondiale de la propriété intellectuelle, mais surtout à l'OMC dans le cadre de l'ADPIC (Aspects des Droits de Propriété Intellectuelle qui touchent au Commerce) et de l'AGCS (Accord Général sur le Commerce et les Services).

Les autoroutes de l'information ou la fracture numérique ? Le niveau des inégalités reste très important dans de nombreux domaines ${ }^{19}$ (Nord/Sud, urbain/rural, jeunes/vieux, accès/débit... ) et abyssal avec les pays les plus pauvres. C'est un dossier dans lequel le rôle du Sénégal a été important à l'issue du sommet SMSI avec le lancement du FSN, le Fond de Solidarité Numérique. Mais deux problèmes se posent : d'une part le fonctionnement multilatéral de ce Fond (avec un statut de droit suisse !) se heurte au traditionnel fonctionnement par projets qui a toujours la préférence des grandes agences de moyens (FMI, Banque Mondiale, Francophonie...), et d'autre part le financement de ce FSN reste soumis à des collectes ou au mécénat de Partenariats Public Privé. Le grand concept de solidarité numérique, qui sous-tend normalement la mise en place de ce Fond, a donc quand même suivi une approche plus libérale (effet bénéfique du marché et des initiatives privées) que publique (engagements et interventions des états).

On peut conclure ce très rapide survol des questions posées par deux remarques, sur l'histoire et sur les lieux du débat entre vision technique et vision sociale d'Internet :

- historiquement la régulation d'Internet associait avant tout des universitaires, des experts privés, des entreprises et des usagers des réseaux. Les questions de normalisation technique ont engendré des phénomènes de grande ampleur qui ont commencé à structurer de nouveaux systèmes de régulation, mais ceci s'est fait par agrégation cumulative et ajustements ex post : les conflits entre le modèle économique de l'Open Source et celui des standards propriétaires, l'autorégulation dans les forums techniques tels que l'IETF ou le W3C, l'intégration verticale des industries de télécommunication et celles des contenus... Au nom de l'intérêt général, les gouvernements sont alors entrés dans la partie avec une ambition régulatrice (notamment à l'occasion des contradictions entre le droit conventionnel des états et des pratiques délictueuses extraterritoriales) pour résoudre les

\footnotetext{
${ }^{18}$ http://www.droit-technologie.org/

${ }^{19}$ Voir « De la fracture numérique aux perspectives numériques » une publication UIT/UNESCO www.orbicma.ca
} 
controverses et les conflits entre les niveaux de territoires (local, national, international) et entre des intérêts sectoriels (Etats, marché, société civile) d'ailleurs eux-mêmes hétérogènes ${ }^{20}$;

- géographiquement les lieux de ces débats apparaissent différents. Les partisans de la vision technique favorisent les comités d'experts, les organismes de normalisation, les consortiums d'industriels ... qui cherchent à prendre en charge les problèmes de normalisation, toile de fond du fonctionnement quotidien d'Internet. Les partisans d'une vision sociale privilégient les forums, les déclarations et les conventions... pour valoriser les expériences locales avec des partenaires de tous horizons. Mais Internet devenant le moteur de la société post-industrielle, le couple technique/politique est indissociable, et la distinction entre une "gouvernance des ressources techniques » et une « gouvernance élargie d'Internet » n'a pas résisté à l'épreuve du réel : l'exemple de l'échec des verrous DRM (Digital Rights Management) sur les fichiers MP3 montre que la normalisation ne concerne pas seulement les fonctionnalités techniques, mais directement le contrôle des usages et la constitution des pouvoirs.

C'est d'ailleurs cet indissociable couplage technique/politique qui impose une recherche de nouveaux fondements théoriques, fondés sur la légitimité et la réflexivité. Un tel travail est nécessaire pour évacuer ce que peut avoir d'apolitique la notion de gouvernance.

\section{A la recherche de fondements théoriques : la légitimité faible et la réflexivité forte}

D'un côté la loi de la jungle (devenue intolérable, depuis qu'Internet est un système sociotechnique pour toute la société), de l'autre côté l'espoir d'une gouvernance mondiale (qui reste impossible, dans la confusion des multiples agences intergouvernementales) et entre les deux une multitude de comités ad hoc (sans pouvoir, quand ils ne représentent qu'eux-mêmes) : il semble bien que le problème de la régulation d'Internet soit mal posé.

Le problème est mal posé au niveau théorique, d'une part si on ne s'appuie que sur la gouvernance au sens de la théorie de l'agence, et d'autre part si on croit que le problème est spécifique à Internet. Car l'échec de la régulation par les « représentants élus » des États et l'émergence de pôles de «spécialistes non élus » concernent bien d'autres domaines : privatisation de la Banque Centrale Européenne, émergence des communautés de communes, développement de la justice privée, etc. La Directive européenne du 8/06/2000 exprime bien cette idée actuelle : «les législations ne doivent pas faire obstacle à l'utilisation des mécanismes de règlements extrajudiciaires des différends ».

\footnotetext{
${ }^{20}$ Par exemple, nombre de pays occidentaux tendent à s'aligner sur le statu quo (sécurité, mais surtout stabilité des intérêts acquis), alors que pour les grands pays à l'économie émergente (Chine, Inde, Brésil...) Internet est considéré un vecteur pour redéfinir le multilatéralisme au profit de nouveaux blocs régionaux.
} 
Il s'agit donc de questions politiques plus fondamentales : comment faire une société avec des libertés ? Comment articuler les sources de normativité ? Comment garantir les deux exigences de l'innovation et de la démocratie ? On peut donc proposer modestement de reposer ici le «problème de la régulation » à l'aune de deux concepts qui ont émergé en sociologie : celui de la légitimation faible (avec notamment les propositions de Jürgen Habermas et de Michel Foucault) (2.1) et celui de la réflexivité forte (avec les propositions de Pierre Bourdieu, d'Ulrick Beck et d'Antony Giddens) (2.2). Ceci permettra d'éclairer ensuite le débat actuel sur la régulation, entre autorégulation, co-régulation et multi-régulation (2.3).

\subsection{La légitimité faible, fondement théorique pour une analyse historique de la gouvernance d'Internet}

L'analyse d'Habermas (2000) est fondée sur la crise de légitimation qui frappe le capitalisme, sur la contradiction éthique entre le capitalisme avancé et la démocratie. Dans le régime traditionnel de légitimation forte, les partenaires d'un Etat-nation pouvaient compter sur leurs expériences antérieures des motivations et des préférences de chacun pour interpréter celles-ci et fonder leur confiance : conscience d'une identité nationale sur un territoire, éducation nationale, pratiques de négociation dans les relations de travail, solidarités forgées dans la division sociale du travail... Ce régime perd aujourd'hui son utilité quand il s'agit de créer la confiance entre différents groupes d'intérêt issus d'horizons radicalement différents, et surtout quand il s'agit de questions nouvelles où la référence à un monde commun est impossible (Internet... mais aussi Ecologie).

Il faut alors créer la confiance sur une autre base, en extrapolant ce monde commun à partir des expériences. La seule voie praticable est de s'accorder sur les procédures collectives, de chercher une confirmation non pas sur la substance des solutions mais sur la garantie de pouvoir poursuivre collectivement la recherche d'autres accords ${ }^{21}$. La légitimité faible peut alors s'élaborer dans ces procédures de négociation, par l'acquisition pratique de nouveaux codes de confiance. La rationalité procédurale vient ici du trajet collectif qui est suivi pour établir son acceptabilité : comment les problèmes sont-ils formulés, comment les solutions sont-elles évaluées... On voit qu'il s'agit d'un dépassement considérable de la rationalité procédurale telle que la proposait Herbert Simon. Le recours à un point de vue moral d'équité redevient possible, dans la mesure où un principe n'est désormais acceptable que s'il est soumis à l'interchangeabilité des rôles et à une réversibilité complète des normes. L'avantage de la légitimation faible est de favoriser le rôle interactif des cultures intermédiaires dans l'élaboration de normes communes (communautés, minorités agissantes, zones

\footnotetext{
${ }^{21}$ L'élaboration des normes sur Internet repose, par exemple, sur la technique du « rough consensus » consistant à procéder par élaboration incrémentale d'une norme qui est réputée adoptée si aucune contre-proposition crédible ne se construit. Cette procédure repose sur des bases très particulières dans la mesure où aucun accord préalable entre les parties prenantes ne définit l'identité de ces dernières, ni leur statut.
} 
hybrides mi-publiques mi-privées...), en valorisant les espaces publics, le débat argumenté, l'apprentissage, la coopération, l'autocontrôle, l'intelligence collective ... Allons-nous vers la constitution d'une conscience cosmopolitique?

Mais, même si cet idéal du modèle délibératif, de l'expérimentalisme démocratique (Maesschalck 2008), du «ménage à trois » Etats/Citoyens/Marché (Lévesque 2004) est en germe dans certains modes de régulation d'Internet, ce processus n'est pas sans sujets. Comment les différents acteurs, de légitimités et de statuts différents, pourraient-ils s'impliquer de la même façon étant donné la complexité et technicité des débats, le coût d'entrée pour les parties prenantes (en terme de coût de collecte d'information, de conditions d'accès à ces informations, de fiabilité des informations, de temps à consacrer...), étant donné la sur-représentation des experts et des intérêts industriels et commerciaux ? Les formes de légitimité propres à la technique (qualité des contributions, implication dans les collectifs...) heurtent de front celle de la légitimité démocratique ou politique.

C'est pourquoi l'analyse de cette crise de légitimité de l'Etat peut bénéficier des apports de Michel Foucault (1978) sur les relations entre pouvoir et société Pour Foucault les grandes luttes contre l'exploitation économique et sociale, qui avaient marqué le XIXe siècle, sont devenues aujourd'hui des luttes à l'égard de micros pouvoirs : un petit chef, un gardien, un directeur, un juge, un vice-président d'université... «L'une des premières choses à comprendre, c'est que le pouvoir n'est pas localisé dans l'appareil d'État, et que rien ne sera changé dans la société si les mécanismes de pouvoir qui fonctionnent en-dehors des appareils d'État, au-dessous d'eux, à côté d'eux, à un niveau beaucoup plus infime, quotidien, ne sont pas modifiés » (Dits et écrits, Tome II, 1975, p. 406). Le concept de «gouvernementalité », formé à partir des termes de gouvernement et de mentalité, désigne cette rencontre «entre les techniques de domination exercées sur les autres et les techniques de soi » (La gouvernementalité, 1978, Dits et écrits, Tome III). L'analyse de Foucault n'est donc pas centrée sur l'Etat mais sur les instruments, les «technologies gouvernementales», qui constituent les individus en sujets moraux (Lascoumes 2004, Meyet et al. 2005). Une technologie gouvernementale est définie très largement comme un instrument à la fois technique et social qui organise les rapports Etat/Individus en fonction des représentations et des significations qu'il crée. Un tel instrument peut être aussi bien une procédure, qu'une observation statistique, une cartographie, un prélèvement fiscal, un règlement, un système incitatif, une contractualisation, une institution, une convention cadre, une norme, un standard, etc. Nous ne sommes pas loin des analyses en termes d'actants et d'acteur-réseau (Callon et Latour 1986), ou de régulation par les outils de gestion (Moisdon 2000), mais ici l'analyse des instruments permet de comprendre les relations entre pouvoir et savoir, entre société politique et société civile : une gouvernementalité spécifique produit un style de vie, qui amène les individus à se concevoir et à se gérer eux-mêmes dans le système considéré, à réagir «normalement », avec discipline mais sans coercition. 
Le concept de gouvernementalité est évidemment central pour analyser la dissuasion de la fraude sur Internet (Berensford 2003), l'invasion des technologies mobiles (Leclercq 2010), le « biopouvoir » sur les corps lié à l'identification biométrique... ou peut-être l'élaboration d'une Charte de l'internet que beaucoup appellent aujourd'hui de leurs vœux. Il permet de relativiser le rôle joué par la technique dans l'émergence des nouvelles pratiques régulatrices, en les resituant dans le cadre d'évolution plus large de l'enchevêtrement de légitimités de natures différentes.

Ces nouveaux régimes de légitimité faible caractérisent les Etats-nations, mais aussi l'éducation ou les organisations traditionnelles. L'entrée dans la société de l'information est une entrée dans une société de la connaissance, où l'accent est mis sur les compétences individuelles mais surtout collectives qui doivent se perpétuer tout au long de la vie. À la légitimité de l'Education nationale (vision où des élèves puisent et s'approprient des informations codifiées dans un processus asymétrique) s'oppose désormais une vision où les connaissances sont collectives et émergentes, incluant des connaissances tacites liées à l'action, et incluant des compétences nouvelles dans l'articulation d'usages multi-modaux. Et la figure traditionnelle du lien communautaire supposant la proximité est modifiée par les nouveaux environnements techno-cognitifs : les modèles de cognition distribuée entre différents acteurs (humains et machines) remettent en cause la rationalité supposée des individus. La question de la légitimité faible se pose aussi dans l'analyse de la relation entre cognition et organisation, avec une légitimation de l'organisation se présentant maintenant comme un véritable système cognitif : représentations collectives des savoirs, modalités d'échange et de codification, groupes de projets flexibles, multi-appartenance à des formes renouvelées de communautés, parcours d'autodidactes, systèmes de recommandation d'experts... Et, à la légitimité traditionnelle des niveaux de centralisation, de l'étendue de contrôle ou du degré de division du travail dans les organisations, il conviendrait sûrement de substituer les notions de degrés de standardisation des procédures ou de modalités de partage de l'information., tant à l'intérieur qu'à l'extérieur de ces organisations.

Dans l'enchevêtrement de légitimités de nature différentes, le processus d'institutionnalisation de la gouvernance d'Internet est marqué par les interactions/confrontations/compromis entre les règles de droit (produites par les Etats et les organisations inter-gouvernementales: lois, conventions, sanctions...) et les nouvelles «petites » institutions qui se créent (par l'action des entreprises et par l'activisme civil : chartres, codes, consortiums, forums...).

Ces petites institutions acquièrent leur légitimité de différentes façons :

- en s'autoproclamant coordinateur et régulateur, un consortium comme IMS peut soumettre à l'épreuve de l'usage et de la concurrence les nouveaux principes pédagogiques qu'il avance, sans avoir besoin de recourir à une reconnaissance a priori de sa légitimité (Fallery 2004);

- en s'affranchissant des contraintes de localisation et de rémunération à court terme, une communauté de développement de logiciel libre travaille sans avoir besoin de la légitimité 
d'une entreprise et favorise ainsi l'émergence de cultures intermédiaires entre production et consommation (Messonier et al. 2010);

- en légitimant progressivement une nouvelle façon de produire et d'accéder au savoir, le phénomène de l'encyclopédie Wikipedia modifie à la fois les propriétés de signification (la langue partagée sur Internet) et les propriétés de domination (la remise en cause des éditeurs et des fournisseurs traditionnels de contenus) (Sanger 2005);

- en tentant (péniblement) de mettre au point son mode de fonctionnement, l'IGF Internet Gouvernance Forum, commence néanmoins à donner une légitimation à la «société civile $»^{22}$.

Ce nouvel agencement, cette création de «l'institution Internet», se déroule dans un cadre juridique et jurisprudentiel faible, avec peu de sanctions légales. La place des règles informelles apparaît comme un élément important du système juridique qui se construit au nom de la gouvernance.

Les stratégies des grandes entreprises sont alors à la fois d'ordre offensif (création de nouveaux marchés, création de nouveaux réseaux ${ }^{23}$ ) et défensif (limitation de l'intervention publique) : elles développent de nouvelles normes de légitimation des TIC (par un travail sur la représentation de « la » société de l'information, de la nouvelle économie, de la société sans conflit...) et de nouvelles justifications de leur rôle social et politique (par les discours sur l'autorégulation par rapport aux Etats). Mais la construction d'ensemble qui résulte de ces stratégies ne peut pas être considérée comme voulue en tant que telle par ces grandes entreprises. Car leur intention rencontre à la fois un tissu réglementaire et institutionnel qui perdure en partie (droit commercial ou droit social, par exemple), un tissu de formes organisationnelles intermédiaires (la constellation ISOC, W3C, IEE par exemple) et un tissu de confrontations dans la mise en oeuvre des nouvelles institutions (la résistance des utilisateurs aux DRM Digital Right Management systems ou la défense du peer-to-peer par exemple). On peut donc parler d'une reconfiguration des rapports de force par une construction dynamique de la légitimité des acteurs et surtout de leurs représentants. Cette dynamique du processus d'institutionnalisation d'Internet permet de donner une vision historique de cette construction, soit vers une accentuation d'une forme libérale de la concurrence (avec le développement d'un Internet de marché, globalisé), soit vers un rééquilibrage démocratique dans la communication (avec le développement d'un Internet hiérarchisé et contextualisé).

\footnotetext{
22 www.voxinternet.org

${ }^{23}$ L'économie des T.I.C. est marquée par la production de biens complexes réalisés grâce à l'assemblage de composants. L'organisation modulaire de cette production engendre de multiples interdépendances (élaboration plus ou moins consensuelle des normes d'interfaces, nouveaux découpages des droits de propriété...). Il en résulte des structures industrielles en réseaux de «coopétition», de nouvelles externalités, des effets de verrouillage...
} 
2.2. La réflexivité forte, fondement théorique pour une analyse politique de la gouvernance d'Internet

Le sens commun du terme réflexivité suggère l'idée de retour sur soi, fondé sur l'introspection puis le passage discursif du tacite à l'explicite. Mais il s'agit aussi d'un processus d'objectivation, d'incorporation du «sens pratique » au sens de P. Bourdieu. Il s'agit enfin de la rétroaction, de la récursivité, qui passe par le mouvement de structuration du social au sens de A. Giddens et U. Beck.

Pour Bourdieu (1992) les agents occupent une position située dans l'espace et dans l'histoire, dans des champs sociaux sous tension. Ce contexte économique, culturel et social détermine des lignes de forces pour les trajectoires et les stratégies des agents, dans l'espace des possibles délimité par les structures du champ (Pailot 1996). Bien que son apport soit fondé sur le concept plutôt déterministe d'Habitus («l'habitus c'est tout ce qu'un individu possède, et qui le fait », « l'habitus se forme d'avoirs qui se transforment en être », «l'habitus a un effet de renforcement en transmuant l'être en devoirêtre » ... citations par Couturier 2002), Bourdieu développe une réflexivité à trois niveaux (Couturier 2002):

- d'abord une réflexivité au sens commun du terme, qui peut se constituer en habitus réflexif (par exemple au niveau des acteurs eux-mêmes dans les débats sur la vie privée, la régulation des contenus, les problèmes des droits de propriété intellectuelle...);

- ensuite une réflexivité comme méthode, essentiellement collective, pour s'émanciper du rapport immédiat au social : c'est l'image qui est renvoyée à un sujet connaissant par d'autres sujets connaissant, et qui engage une véritable réflexivité (par exemple les analyses sociologiques des communautés Open Source, ou de l'encyclopédie Wikipedia...);

- enfin une réflexivité plus institutionnelle, qui permettrait de développer le concept de « champ social » des TIC, fondamental ici pour une théorie de la régulation : le rapport est dialectique, réflexif, entre les structures objectivées et les dispositions individuelles par lesquelles ces structures s'actualisent (par exemple dans les processus d'apprentissage organisationnel qui se développent au sein des commissions d'éthique, ou dans les associations professionnelles internationales).

Giddens (1987) pose plus radicalement la question de la réflexivité, en en faisant la base d'une théorie de la structuration. La réflexivité caractérise ici la boucle récursive entre les interactions quotidiennes (relations situées, choisies, explicitables, et où se déroulent des jeux de communication, de pouvoir et de moralité/sanction), et les formes structurelles des pratiques sociales stabilisées (avec leurs trois propriétés : de signification sémantique, de domination des ressources et de légitimation morale). Ces propriétés structurelles guident et contraignent les interactions quotidiennes des acteurs, 
tout en étant actualisées par ces interactions : c'est la «dualité du structurel ». La reproduction de l'ordre social est assurée par la médiation de routines évolutives (compétences, rôles, métiers...). Un jeu s'organise donc entre une appropriation flexible et potentielle par les individus et les contradictions successives entre les différents systèmes sociaux (ici les Communautés, la Technologie, les Organisations) : la construction « réflexive » de Wikipedia est un bon exemple.

L'interaction quotidienne située relève à la fois du contrôle réflexif du contexte (sur soi et sur les autres), de la rationalisation (conscience théorique et discursive du fondement des activités) et de la motivation (désirs inconscients qui inspirent le potentiel d'action). Tous les individus ont ainsi à la fois une connaissance remarquable des conditions et conséquences de ce qu'ils font dans leur vie de tous les jours (le déroulement d'un Chat sur Internet, par exemple), mais aussi une méconnaissance des conditions non reconnues de l'action et des conséquences non intentionnelles de cette action sur les systèmes sociaux (la construction évolutive de la Netiquette pendant le déroulement du Chat, par exemple).

La thèse de la «modernisation réflexive », développé par Beck, Giddens et Lash (Beck et al. 1994) considère la confrontation réflexive de la société industrielle avec elle-même. Les individus ne prennent plus les savoirs « savants » pour argent comptant, ils n'hésitent plus à contester les experts scientifiques (Sida, nucléaire, OGM, écologie, nanotechnologies..) et s'approprient des capacités d'expertises «profanes ». Pour Internet, les grandes prophéties liées au progrès, à l'emploi ou à la croissance ont éclaté après la bulle spéculative des années 2000. Les effets pervers de la politique industrielle étant dévoilés, le projet technocratique étant reconnu comme un projet politique associé à la doctrine économique du laisser-faire, il peut être soumis à une critique politique et éthique : le voile qui fétichise les décisions politiques comme des décisions techniques est déchiré (Beck et al. 1994). Un nouvel espace public de contestation de la «pensée unique» se développe. Comme pour l'écologie, nous ne sommes plus dans un univers du risque (avec calcul des probabilités) ni même dans un univers incertain, mais dans un univers controversé (Godard 1999) : perceptions différenciées des problèmes, représentations différentes des intérêts, réversibilités différentes des phénomènes, stabilisation différenciée de la connaissance scientifique. "La première vague de dérégulations nationales exige une seconde vague de re-régulations transnationales. Sans ce pas décisif vers une démocratisation cosmopolite, nous nous dirigeons vers une société mondiale technocratique et postdémocratique » (Beck, cité par Vandenberghe 2001). Beck propose donc, comme Habermas, une politique systématique de la «table ronde » réunissant des citoyens, des experts et des contre-experts et des élus.

Mais cette «modernisation réflexive » se conjugue aussi à une «individualisation réflexive » (Beck 2003). Dans les conditions de la modernité avancée (pluralité des mondes vécus et des styles de 
vie), les individus sont libérés non seulement des contraintes culturelles (religion, tradition, scientisme...), mais aussi des contraintes structurelles (la classe, le statut, la nation, le genre, la famille...). Ils peuvent, ils doivent, réfléchir librement à la vie qu'ils veulent mener. L'identité personnelle devient alors une opération réflexive, une décision consciente de choisir et façonner sa propre biographie. L'individualisation est réflexive pour trois raisons. Premièrement parce que cette action quotidienne, qui se libère de la culture et de la structure (éducation, travail, frontières, Etats...) n'a pourtant pas lieu dans un vide social (globalisation culturelle, insécurité du marché de l'emploi, communautés, mouvements sociaux transnationaux...). Deuxièmement parce que les individus et les groupes, qui s'approprient ou non les technologies en les mettant au service de leurs besoins d'identité, participent ainsi à la transformation de ces institutions (sociabilité téléphonique, livre numérique...). Enfin l'individualisation est aussi réflexive parce qu'il n'y a pas de globalisation globale, il n'y a pas de globalisme. Il y a seulement une globalisation multidimensionnelle « qui a lieu localement et qui change le local »(Beck, cité par Vandenberghe 2001): même MacDonald, Madonna, le rap ou la pizza ... nécessitent toujours une traduction et une interprétation locale. Les cultures s'interconnectent, entrent en fusion, et si une nouvelle culture émerge, elle est hybride, cosmopolite, créolisée : une pluralité sans unité.

\subsection{Auto-régulation, co-régulation ...ou multi-régulation ?}

Comment gouverner sans gouvernement? Ou plutôt comment gouverner en régime de légitimité faible ? La gouvernement d'Internet se présente ici comme un modèle politique à construire sur les thèmes de la responsabilité et de la transparence, de la participation à la prise de décisions, de la gestion des affaires publiques et de l'implication de la société civile. L'analyse de la privatisation proposée par Pesqueux (2007) montre comment la régulation par les instruments «efficaces » de la gouvernance des entreprises (issus du libéralisme économique et du positivisme scientifique) s'imposent aujourd'hui dans la sphère publique avec le New public management (en assouplissant la rigueur de la loi et généralisant les règles et les normes acceptées par les acteurs eux-mêmes). Le projet de la privatisation est finalement de «désinstitutionnaliser les institutions », c'est-à-dire de les considérer comme des organisations comme les autres, au nom de l'efficacité socio-économique, de la qualité de service rendu et de l'efficacité de gestion. Mais en même temps que les grandes institutions se désinstitutionnalisent, les petites organisations s'institutionnalisent, avec notamment le statut de parties prenantes qu'ont maintenant les organisations non gouvernementales ou les consortiums privés-publics dans les délibérations internationales.

Légitimation plus ou moins faible et réflexivité plus ou moins forte se conjuguent alors pour faire avancer trois grands modèles différents de la régulation (Berleur et al. 2001, Dedeurwaerdere et Maesschalck 2001, Marzouki et Méadel 2004). Ces trois modèles cohabitent bien sûr aujourd'hui dans 
Internet : il y a de l'autorégulation libertaire, il y a de la co-régulation normative, et il y a de la multirégulation organisée.

\section{Le modèle libéral-libertaire de l'autorégulation}

Le modèle de l'autorégulation est celui de la naissance d'Internet, celui d'une recherche collaborative entre universitaires et industriels, celui aussi d'une vision libertaire de l'activité économique. Dans l'autorégulation, le préfixe auto désigne un degré variable de contraintes collectives, relativement bien établies et reconnues, mais n'émanant pas directement des états : autorégulation «spontanée»par des normes privées dans des communautés comme le W3C, ou autorégulation «déléguée » dans le cas de l'ICANN. L'autorégulation apparaît donc comme une technique juridique de création de règles, mais élaborées par les destinataires eux-mêmes de ces règles. Les défenseurs de l'autorégulation soutiennent une réflexivité d'ajustements routiniers, un ajustement de type fonctionnel. Ces réseaux d'acteurs autorégulés pourraient ainsi aller au-delà du comportement marchand individuel, tout en développant un mode d'action collective différent de celui classiquement organisé par les états (Ogus 1995).

Mais Internet n'est plus un réseau de pionniers et la donne a bien changé :

- les règles d'autodiscipline sont finalement peu contraignantes (Netiquette, chartes de bonne conduite) et surtout toujours susceptibles d'être transgressées en fonction d'intérêts très divergents ;

- les objectifs des industriels sont aujourd'hui de purger Internet de tous les obstacles à la création de nouveaux marchés (commerce électronique, éducation en ligne, édition numérique... ${ }^{24}$ ), en cherchant à cantonner l'intervention publique à la protection d'intérêts particuliers (qui sont d'ailleurs très hétérogènes: racisme, pornographie, diffamation, criminalité ....);

- le mode d'élaboration des standards techniques d'Internet peut rester fondé sur la recherche $\mathrm{du}$ 《 consensus approché » (rough consensus)... sauf quand les enjeux de pouvoir deviennent très importants pour les sociétés multinationales ou les états (comme dans le cas des droits de propriété, ou dans celui de la sécurité).

Le modèle de l'autorégulation est donc largement aujourd'hui celui des procédures du marché, avec un risque renouvelé de domination.

\section{Le modèle institutionnel de la co-régulation}

\footnotetext{
${ }^{24}$ Americas Business Forum, World Business Council for Sustainable Development, Global Information infrastructure Commission, Global Business Dialogue for e-commerce...
} 
Le modèle de co-régulation affirme le maintien du rôle de l'Etat, à côté (mais non pas audessus) d'une multitude d'acteurs définissant des régulations et les mettant en œuvre. La co-régulation se veut une voie médiane entre trop d'Etat et trop de liberté, la société étant confrontée à deux risques nouveaux : celui d'un Internet « hors des lois » et celui d'un Internet uniquement marchand.

Le Forum des Droits sur l'Internet (FDI) ${ }^{25}$ est par exemple une association française créée et soutenue par le gouvernement, avec pour ambition de «favoriser l'adaptation de l'ensemble du droit et des pratiques au contexte nouveau de la société de l'information » et d' «associer l'ensemble des acteurs à la construction de la civilité de l'internet »: information du public, débats en ligne, production de rapports pour les politiques publiques...

La co-régulation ne se limite donc pas à la réglementation (paiement électronique, signature électronique...), mais elle correspond plutôt à une forme d'intervention publique normative. On notera pourtant que le choix d'une association loi 1901 pour aborder des enjeux d'intérêt public montre l'extrême discrétion des pouvoirs publics. Et comme Internet n'a jamais été un réseau d'enfants de chœur, les obstacles à une co-régulation négociée sont évidents :

- limites du volontariat et de l'expertise des parties en présence, ce qui entraîne une surreprésentation des experts et des intérêts industriels et commerciaux ;

- poids élevé des pratiques marchandes par rapport aux recommandations publiques ;

- fragmentation de l'espace public, qui ne peut qu'institutionnaliser des intérêts qui sont finalement assez divergents (par exemple dans la loi Création et Internet et la haute autorité Hadopi) ;

- instrumentalisation possible de la part des hommes politiques comme de celle des lobbyings ;

- espace resté très européen de cette co-régulation (cogestion ?) en face d'un espace mondial du réseau ;

et surtout ...

- absence de vrais débats, dans la co-régulation, sur les protocoles et standards techniques d'Internet, au nom de l'efficacité technologique. Alors que la normalisation s'opère largement expost et de façon cumulative, sous la pression des industriels, des prestataires de service... mais aussi des utilisateurs (téléphonie IP, copie privée...).

Les défenseurs de la co-régulation cherchent à valoriser une capacité réflexive par les processus d'apprentissage adaptatif dans des structures institutionnelles d'incitation soutenues par les Etats. Dont acte. Mais dans ces structures cette réflexivité n'est-elle pas uniquement rétrospective, cherchant du sens dans certaines traditions existantes (le commerce, les langues, le livre papier..), mais en fait incapable d'anticiper de nouveaux contextes, contextes et usages en évolution permanente dans le brouillement créatif, celui des ingénieurs, des managers et des usagers ?

\footnotetext{
${ }^{25}$ http://www.foruminternet.org/
} 
Le modèle de la co-régulation est donc celui d'une régulation normative selon les procédures de la démocratie, généreuse utopie mais qui fait sans doute l'impasse sur le pouvoir réel des différents acteurs.

\section{Le modèle hiérarchisé de la multi-régulation}

Les insuffisances d'une autorégulation purement technique et l'inefficacité de la co-régulation au sens démocratique du terme nécessiteraient donc d'évoluer vers des modes de gouvernance plus réflexifs dans un cadre de légitimation faible. Nous avons montré qu'on pouvaient disposer des fondements théoriques nécessaires, bien au delà d'une vision simplement contractuelle même élargie aux parties prenantes. Au niveau pratique ceci amène une réflexion autour d'un principe de hiérarchie, non pas dans les institutions, mais dans la conception même de la régulation. Plutôt qu'une «cointervention sur pied d'égalité de l'Etat, des entreprises et des groupes d'intérêt dans les procédures de régulation »(Brousseau 2001, p. 26), Brousseau propose cette hiérarchie « réflexive » entre différents sous-systèmes de normativité :

- avec, à un premier niveau, des cadres institutionnels «privés », qui peuvent élaborer leurs solutions collectives de coordination, sanctions comprises, dans les trois champs spécifiques (infrastructures du réseau, grands services sur Internet, et applications pratiques ) : il s'agit à ce niveau de favoriser l'interaction récurrente, l'auto-évaluation, les compétences collectives, les certifications... mais aussi la culture d'usage des acteurs, la réversibilité dans l'ajustement routinier, les consensus approchés...

- et, à un deuxième niveau, une instance ultime de régulation qui doit pouvoir régler les conflits entre ces normes privées et locales : encadrement institutionnel, mesure des externalités liées aux comportements de chaque communauté, culture politique du bien commun visant à maintenir l'ouverture du réseau face aux détournements possibles, asymétrie du collectif par rapport aux intérêts particuliers, enjeux éthiques à venir qui pourraient mettre en cause la communauté humaine ...

Prenons un exemple. On voit bien qu'un choix politique au niveau du bien commun (par exemple une politique de droits de propriété en matière culturelle) a des implications différentes pour des acteurs différents (d'un côté les producteurs de media commerciaux, et d'un autre côté les membres de la communauté universitaire). Il s'agirait donc ici, dans la cadre d'une multi-régulation hiérarchisée :

- au niveau de l'instance ultime, de mettre en avant un principe, celui de l'accès le plus large aux ressources du réseau. Ce serait le rôle d'une sorte de «superintendant du réseau », pour assurer à la fois la distribution initiale des droits de propriété et le renforcement des accords qui régulent Internet (ceci n'est pas complètement utopique: dans un autre problème, celui des noms de domaines, une évolution négociée de la représentativité au sein de l'ICANN pourrait peut-être légitimer son rôle de superintendant); 
- à des niveaux plus spécifiques et locaux, de favoriser la diversification des producteurs d'information, à travers des dispositifs d'ordre matériel ou légal qu'ils feront émerger eux-mêmes (en prenant appui sur les communautés à travers lesquelles ils communiquent: dans notre exemple des droits de propriété, le réseau « Couperin » joue un rôle de négociation et d'expertise des ressources documentaires électroniques).

Dans cette forme hiérarchisée de la multi-régulation le rôle d'une instance de régulation ultime ne serait pas de sanctionner juridiquement, mais de favoriser des mécanismes incitatifs pour une réflexivité durable et une capacité d'anticiper de nouvelles formes de coopération. Il s'agit d'une certaine façon de faire fonctionner à la fois l'autorégulation libertaire et la co-régulation institutionnelle, en fonction des questions, des besoins et des niveaux.

Mais ceci devra passer par une «culture intermédiaire » de la régulation, notamment grâce à la formation et donc à la formation de formateurs : une transformation croisée entre une culture d'usage (par agrégation des expériences) et une culture politique (par la définition de biens publics).

\section{Conclusion}

A travers un débat opposant une Société de l'information à des Société des savoirs, cet article avait pour première ambition d'exposer certains enjeux d'Internet, notamment la gestion des noms de domaines, la tarification étagée, le contrôle des contenus, l'exception culturelle, la propriété intellectuelle et la fracture numérique. Nous avons montré qu'il n'y a pas d'un côté des questions techniques et d'un autre côté des questions sociales. Il s'agit toujours de questions politiques fondamentales sur la normativité, l'innovation, la démocratie, les libertés.

Posant alors la question de la régulation d'Internet (autorégulation, co-régulation, multirégulation ?), la deuxième partie avait pour ambition de proposer les fondements théoriques nécessaires pour décrire et analyser les problèmes de confiance et légitimité, de technologies gouvernementales, d'institutionnalisation, de modernité... Cela a été fait en référence à deux concepts : la légitimation faible, développée notamment par J. Habermas et M. Foucault, et la réflexivité forte, développée notamment par U. Beck et A. Giddens. Un jeu véritable social réflexif devrait pouvoir s'accélérer entre d'une part une appropriation potentielle par les individus et d'autre part les contradictions successives entre différentes formes structurelles à légitimité faible (les communautés, la technologie, les organisations, les entreprises, les Etats, le marché).

Le premier résultat de notre analyse est donc d'ordre théorique : la vision contractualiste standard d'une simple "gouvernance », même élargie aux parties prenantes, ne permettra pas de rendre compte de la nouvelle régulation qui se construit pour Internet. Car il s'agit moins d'optimiser la gestion des conflits à régler (normalisation, qualité de service, sécurité et résilience, droits de 
propriété...) que de favoriser une nouvelle forme d'institutionnalisation dont les répercussions dépassent largement le réseau lui-même (libertés, cultures, identité numérique, biens publics mondiaux...). Ce qui est en cause c'est donc moins une nouvelle forme de contrôle ou d'autocontrôle qu'une nouvelle forme d'apprentissage inter-organisationnel, un équilibre entre ordre et désordre, pour préserver la capacité d'adaptation d'Internet, la diversité des utilisateurs et des usages, et ainsi le plus grand potentiel d'innovation de l'histoire récente. Et c'est pourquoi la discipline des Systèmes d'Information peut (doit) apporter sa contribution, pour mettre en évidence les conditions dans lesquelles des compétences collectives et des transformations institutionnelles peuvent émerger : ceci concerne aussi bien nos apports sur les solutions collectives de coordination (ajustements routiniers, supervision, formes de standardisation, décision collective, évaluation des performances, autoorganisation...) que ceux sur les conditions de l'apprentissage organisationnel et inter-organisationnel (gestion des connaissances, auto-évaluation, formes de confiance, stratégies discursives, coopétition, usages et détournements..).

Le deuxième résultat de notre analyse est d'ordre plus pratique et on peut maintenant tenter de décrire deux grands scénarios de régulation, respectant chacun les principes de l'expérimentalisme fort et de la légitimation faible. Le modèle qui se mettra en place restera probablement aussi «incontrôlable » que le réseau lui-même, c'est sans doute frustrant mais c'est aussi le prix de la liberté. Pour préserver ce potentiel d'innovation d'Internet il faut envisager toute transformation avec prudence, nous parlerons donc plutôt de deux évolutions : vers plus de démocratie directe ou vers plus de démocratie représentative.

Le premier scénario pourrait s'appeler «une évolution de l'autorégulation, vers plus de démocratie directe ». Il s'agit ici de partir de l'esprit général de l'autorégulation, esprit de départ d'Internet lié à sa topologie décentralisée, pour favoriser aujourd'hui une évolution vers un maximum de cercles (ou pôles, ou constellations) constitués autour de régions, de problématiques ou de zones d'expertises. Pour contrecarrer les risques évidents de l'autorégulation (domination par le marché, domination par l'administration américaine...) et puisque l'on craint ici les représentants de grands «machins » internationaux, il faudra alors aller vers plus de démocratie directe, dans ce qui doit toujours rester un «meta-projet », où les nouveaux venus ont la parole et où les mandataires sont révocables. Il ne s'agit pas ici d'organisation pyramidale ni même en cercles concentriques, mais de cercles en intersections multiples. Chacun de ces cercles deviendra une «petite » institution, qui devra se légitimer d'abord par une amélioration de la représentativité et de la transparence en interne, chez tous ses participants (par exemple à l'ICANN en transformant vraiment le Governmental Advisory Commitee en forum transparent). Cette légitimation devra passer ensuite par une amélioration des conditions de la démocratie directe (par consensus avec les fameux RFC Request for comments, mais aussi par forum, par vote, par referendum, par pétition...). Mais dans ce scénario la principale difficulté restera de relier entre eux, en l'absence d'autorité supérieure, ces cercles homogènes spécialisés mais aussi interdépendants dans les aspects très horizontaux d'Internet. A l'intersection de 
ces cercles (qui peuvent être grands : rien qu'à elle, l'ISOC regroupe 28.000 personnes venant de 170 pays, plus de 80 organisations et 90 filiales réparties dans le monde) il faudra donc favoriser le dialogue grâce notamment à la double appartenance des individus à plusieurs cercles, mais sans doute aussi grâce à la référence aux principes communs constituant une chartre à venir pour Internet. On voit qu'au delà d'une cartographie encore à faire de tous ces cercles en intersections multiples, il s'agit bien d'une nouvelle culture intermédiaire à créer, une culture de la solidarité et de la sécurité collective, seule capable de justifier une délégation consentie à chacun de ces cercles.

Le deuxième scénario pourrait s'appeler « une évolution de la multi-régulation, vers plus de démocratie représentative ». Il s'agit ici de partir de l'esprit général de la multi-régulation, celui d'une régulation multipolaire organisée, pour favoriser une évolution vers une différentiation ordonnée en différents niveaux, suivant les principes de subsidiarité, de proportionnalité et de complémentarité des règles. D'une certaine façon cette vision est complémentaire de la première, mais pour éviter que les autorégulations ne soient détournées par des groupes d'intérêt particuliers, elle considère comme nécessaire la définition de plusieurs «étages » de ces cercles en intersections. Dans ce scénario de hiérarchisation ordonnée, il faudra d'abord passer par plus de démocratie représentative dans chacun des cercles, par exemple des élections au futur IFG européen (comme cela se fait chez d'autres acteurs, comme Wikipedia où l'élection des «administrateurs, bureaucrates et développeurs » permet d'assurer notamment les fonctions de police par blocage et de justice par des comités d'arbitrage). Mais la principale difficulté restera ici la légitimation d'un étage ultime, un superintendant d'Internet, une instance fédérale élue qui aura la charge de la cohérence globale et du respect des droits fondamentaux, garante des trois grands principes d'interopérabilité, d'ouverture et de neutralité. Cette instance ultime «d'inter-régulation », indépendante des Etats Unis aussi bien que de l'ONU, reste à légitimer :: un International Internet Council, issu des différents niveaux l'ISOC ? Un Forum mondial de gouvernance d'Internet, issu des différents niveaux d'IGF ? On voit qu'au delà d'une pyramide encore à dessiner, il s'agit là aussi de cette nouvelle culture intermédiaire à créer, une culture de la solidarité et de la sécurité collective, seule capable de justifier ici une subordination consentie, à chacun des différents étages de la hiérarchie.

\section{Bibliographie}

Benamrane D., Jaffré B. et Verschave F.X., (2005), Télécommunications entre biens publics et marchandises, Editions Charles Léopold Mayer, novembre 2005

Benhamou B. (2007), «Les nouveaux enjeux de la gouvernance de l'internet» Regards sur l'Actualité, La Documentation Française, janvier 2007

Beck U., (2003) La Société du risque, sur la voie d'une autre modernité, Flammarion, 2003

Beck U., Giddens A., Lash S. (1994), Reflexive Modernization. Politics, Tradition and Aesthetics in the Modern Social Order, Cambridge, Polity Press.

Berensford D (2003), « Foucault's theory and the deterrence of Internet fraud », in Administration \& Society, vol. $35 \mathrm{n}^{\circ} 1$, mars 2003. 
Berleur J., Ewbank de Wespin T., (2001), «Gouvernance de l’Internet, réglementation, autorégulation, corégulation », Actes du colloque CREIS 2001, en ligne

Bournois F., Duval-Hamel J., Roussillon S., Scaringella J.-L. (2007), Comités exécutifs, Voyage au coeur de la dirigeance, Eyrolles, Paris,

Brousseau E. (2001), «Régulation de l'Internet : L'autorégulation nécessite-t-elle un cadre institutionnel ?» Revue Economique, Septembre 2001

Bourdieu P. et Wacquant L. (1992), Réponses. Pour une anthropologie réflexive, Paris, Seuil.

Callon M., Latour B. (1986), «Les paradoxes de la modernité. Comment concevoir les innovations? », Prospective et santé, 36.

Charreaux G., Schatt A. (2005), «La recherche française en gouvernance d'entreprise : un panorama », Cahier du FARGO n 1050901, Septembre 2005

Couturier, Y. (2002), «Les réflexivités de l'oeuvre théorique de Bourdieu: entre méthode et théorie de la pratique », Esprit critique, vol.04 no.03, Mars 2002

Dedeurwaerdere T., Maesschalck M. (2001), « Autorégulation, éthique procédurale et gouvernance de la société de l'information », Les carnets du centre de philosophie du droit n ${ }^{\circ} 91,2001$

Fallery B. (2004), «Les trois visions de la formation ouverte et leurs propositions de normes: Standardiser les contenus, les activités ou les interfaces? » Revue SIM 2004 n4, vol 9

Foucault M.(1978), La gouvernementalité, Dits et Ecrits : tome III, texte $n^{\circ} 239$. NRF Gallimard

Giddens, A. (1987), La constitution de la société, Paris, Presses universitaires 1987

Godard O. (1999), « De l'usage du principe de précaution en univers controversé », Futuribles, (239240), février-mars, pp. 37-60.

Habermas J. (2000), Après l'Etat-nation, une nouvelle constellation politique, Fayard, Paris.

Leclercq-Vandelannoitte A. (2010), «Un regard critique sur l'approche structurationniste en SI : Une comparaison avec l'approche foucaldienne », Revue SIM 2010, Vol. 15, No 1

Lascoumes P. (2004), «La Gouvernementalité : de la critique de l'État aux technologies du pouvoir », Le Portique $\mathrm{n}^{\circ}$ 13-14, 2004.

Lenoble J. (2005), Théorie de la norme et gouvernance démocratique, Synthèse 2002-2005, Centre de philosophie du Droit (UCLouvain)

Lévesque B. (2004), «Un nouveau paradigme de gouvernance : la relation autorité publique / marché / société civile pour la cohésion sociale », Forum Finance solidaire et consommation responsable, Strasbourg.

Mattelard A. (2003), Histoire de la société de l'information, La Découverte, Paris, 2003, et http://www.monde-diplomatique.fr/2003/08/mattelart/10308 - août 2003

Maesschalck M. (2008), « Droit et capacitation des acteurs sociaux. La question politique de l'application des normes » Revue de philosophie politique de l’Ulg, №1, Décembre 2008

Martinet A.C, (2008) «Gouvernance et management stratégique. Une nouvelle science morale et Politique », Revue française de gestion, 2008/3 - n 183

Marzouki M., Méadel C., (2004), « De l'organisation des nouveaux collectifs à l'organisation de la cité : gouvernance technique et gouvernement politique d'Internet », École des Mines de Paris/CNRS.

Marzouki M., Méadel C. (2004 b) «Francophonie et gouvernance d'internet » in Francophonie et Mondialisation HERMÈS, 2004 pp 228-232 
Meyet S, Naves MC, Ribémont T, (2005), «Travailler avec Foucault: retours sur le politique », L'Harmattan

Meissonier R., Bourdon I., Houze E. (2010) «Comprendre les motivations des développeurs de l'open source à partir de leur participation », Systèmes d'Informations et Management , 2010, vol 15(2).

Moisdon J.C, (2000) "Quelle est la valeur de ton point ISA? Nouveaux outils de gestion et de régulation dans le système hospitalier français », Sociologie du Travail, Volume 42, Issue 1, pp 31-49

Mueller M. (2008), "Info-communism? Ownership and freedom in the digital economy", First Monday, Volume 13, Number 4 - 7 April 2008

Ogus A. (1995), Rethinking self-regulation, Oxford Journal of Legal Studies, 1995 15(1) p 97-108

Pailot P. (1996) «La théorie de l'habitus de Pierre Bourdieu et la psychologie dynamique d'inspiration psychanalytique : quels apports pour la théorie de la rationalité?», Vème Conférence A.I.M.S., Lille.

Peha, J., Lehr, W., \& Wilkie, S. (2007) “The State of the Debate on Network Neutrality”, International Journal of Communication, Aug 9, http://ijoc.org/ojs/index.php/ijoc/article/view/192/100

Pérez R. (2003), La gouvernance de l'entreprise, La Découverte, coll. Repères, 2003.

Pesqueux Y. (2007), Gouvernance et privatisation, P.U.F., Collection La politique éclatée.

Pesqueux Y. (2008), «De la Corporate Governance à la gouvernance organisationnelle», Colloque Fiscalité, droit, gestion, Hammamet, Tunisie

Rioux M. (2004), «Mythes et limites de la gouvernance globale des télécommunications », A contrario 2004/2, Vol. 2

Sanger L. (2005), "The early history of Nupedia and Wikipedia: a memoir" in Open sources 2.0: the continuing evolution, DiBona C., Stone M., Cooper D. eds. O'Reilly Media 2005

Vandenberghe F. (2001), «Introduction à la sociologie (cosmo)politique du risque d'Ulrich Beck» Revue du MAUSS $1 / 2001, \mathrm{n}^{\circ} 17$

Vivant M., (2000) «Propriété intellectuelle et nouvelles technologies. A la recherche d'un nouveau paradigme », Conférence à l'Université de tous les savoirs 2000.

$\underline{w w w . f r e e s c a p e . e u . o r g / b i b l i o / a r t i c l e . p h p 3 ? i d \_a r t i c l e=21 ~}$

\section{Sources consultées en 2010 sur la gouvernance d'Internet :}

http://www.internetgovernance.org/publications.html

www.vecam.org/

http://csdptt.org/

http://www.droit-technologie.org/

www.netgouvernance.org

www.voxinternet.org

www.societesdelinformation.net/

$\underline{\text { http://smsi.internet.gouv.fr }}$

http://www.itu.int/wsis/index-fr.html

http://www.intgovforum.org/cms/ 